\title{
Evaluación externa de los cambios funcionales y la marcha tras una sesión de miofibrotomía múltiple en escolares con diplejía espástica
}

\author{
David Gómez-Andrés, Irene Pulido-Valdeolivas, Juan Andrés Martín-Gonzalo, Javier López-López, \\ Ignacio Martínez-Caballero, Enrique Gómez-Barrena, Estrella Rausell
}

Introducción. Unos 23.000 pacientes con espasticidad han optado voluntariamente por la miofibrotomía múltiple (MFM), una técnica alternativa consistente en seccionar tejidos blandos para liberar restricciones articulares. Iniciada por Ulzibat (Rusia), se realiza fuera de controles clínicos ortodoxos.

Objetivo. Evaluar externamente los efectos de la MFM sobre la funcionalidad motora y la marcha.

Pacientes y métodos. Estudio observacional autocontrolado que evalúa cambios en variables funcionales -Gross Motor Function Classification System, dimensión E de la Gross Motor Function Measure (GMFM) y Functional Mobility Scale- y 32 parámetros de marcha (medidos mediante análisis instrumental) en 22 escolares con diplejía espástica (edad mediana: 9 años y 6 meses; Q1-Q3: 7 años y 11 meses a 11 años y 6 meses) cuyos padres optaron por una MFM (mediana de observación: 4 meses; rango: 3-7 meses).

Resultados. El análisis de conglomerados jerárquicos utilizado para determinar patrones topográficos de cortes en los pacientes detectó que los cirujanos aplicaban a cada paciente uno de tres conjuntos de cortes. Analizados los tres grupos de pacientes, se observó que un grupo empeoró globalmente y una mejora significativa general en la dimensión E del GMFM (diferencia de mediana: 4,86\%; intervalo de confianza al 95\% = 0-6,94\%) tras la MFM. Algunos parámetros de la marcha se normalizaron significativamente (izquierda: rango flexión-cadera, máxima dorsiflexión en apoyo; derecha: velocidad, rotación pélvica media en apoyo y máxima dorsiflexión en apoyo).

Conclusiones. Estos resultados no apoyan ni justifican el uso de la MFM como opción para el tratamiento de la espasticidad. Insistimos en que esta técnica debe evitarse fuera de protocolos de estudio. Sin embargo, los resultados asientan una base objetiva para justificar la realización de un ensayo clínico y estudios observacionales a largo plazo.

Palabras clave. Cirugía. Marcha. Parálisis cerebral infantil. Ulzibat.

\section{Introducción y objetivos}

La parálisis cerebral infantil (PCI) es la causa de discapacidad infantil más común [1]. Gracias a la incorporación de varios tipos de tratamientos personalizados y basados en pruebas científicas, estos niños han podido mejorar su calidad de vida y funcionalidad [2,3]. Sin embargo, estas técnicas no consiguen eliminar completamente las limitaciones, lo que ha favorecido la aparición de un creciente número de terapias alternativas sin el respaldo de estudios que aseguren su eficacia y justifiquen su demanda por parte de los familiares [4-7].

Una de estas terapias alternativas es la miofibrotenotomía o miofibrotomía múltiple (MFM), que Valeri Borisovich Ulzibat desarrolló en Rusia en la época soviética como un 'método para tratar el dolor miofascial' [8]. La MFM es una técnica multinivel que actúa cortando los tejidos blandos involu- crados en las restricciones articulares y, según sus practicantes, 'puede utilizarse para el tratamiento de la espasticidad en la parálisis cerebral y otras enfermedades' [9]. Los cortes de la MFM son percutáneos y se realizan con un bisturí especial en punta de flecha [10] sobre tendones, músculos o fascias. La selección de los puntos de actuación se basa en la 'tensión' subjetiva del músculo o del tendón tras palpación por el cirujano, en el rango de movimiento pasivo o en el dolor detectado tras la compresión de 'puntos dolorosos'. La profundidad del corte se decide en función del aumento que se desea en el rango del movimiento y por la aparición de un 'crujido característico al cortar'. Normalmente no se intervienen más de 20 puntos por acto quirúrgico, que suele durar 15 minutos. Tras la cirugía, el paciente recibe el alta en ese mismo día, se le entrega un informe donde se indica en un mapa estandarizado la posición de los cortes y se le instruye para
Departamento de Anatomía, Histología y Neurociencia; Universidad Autónoma de Madrid, UAM (D. Gómez-Andrés, I. PulidoValdeolivas, J.A. Martín-Gonzalo, J. López-López, E. Rausell). Escuela de Fisioterapia de la ONCE; UAM (J.A. Martín-Gonzalo). Sección de Rehabilitación; Hospital Universitario Infanta Sofía; San Sebastián de los Reyes (J. LópezLópez). Servicio de Cirugía Ortopédica y Traumatología Infantil; Hospital Universitario Niño Jesús; UAM (I. Martínez-Caballero). Servicio de Neurología Infantil (D. GómezAndrés); Servicio de Neurología (I. Pulido-Valdeolivas); Servicio de Cirugía Ortopédica y Traumatología (E. Gómez-Barrena); Hospital Universitario La Paz; UAM. Grupo TRADESMA; IdiPaz (D. GómezAndrés, I. Pulido-Valdeolivas, J.A. Martín-Gonzalo, E. Rausell). Madrid, España.

Correspondencia: Dra. Estrella Rausell Tamayo. Departamento de Anatomía, Histología y Neurociencia. Universidad Autónoma de Madrid. Arzobispo Morcillo, 4. E-28029 Madrid.

Fax:

+34 914975338 .

E-mail:

estrella.rausell@uam.es

Nota:

D.G.A. e I.P.V. contribuyeron ambos como primeros autores al presente trabajo.

Agradecimientos: Queremos agradecer el apoyo de la Escuela Universitaria de Fisioterapia de la ONCE-UAM y de la Agencia de Evaluación de Tecnologías Sanitarias. Gracias también a pacientes, familias, médicos y asociaciones que colaboraron en la realización de este estudio.

Financiación: Financiado por AETES (PI05/90123). 
Aceptado tras revisión externa:

13.01.14

Cómo citar este artículo:

Gómez-Andrés D, PulidoValdeolivas I, Martín-Gonzalo JA López-López J, Martínez-Caballero I, Gómez-Barrena E, et al. Evaluación externa de los cambios funcionales

y la marcha tras una sesión de miofibrotomía múltiple en escolares con diplejía espástica. Rev Neurol 2014; 58: 247-54

(C) 2014 Revista de Neurología permanecer en reposo en cama una semana y para esperar un mes a restaurar la fisioterapia. Los cirujanos suelen recomendar a sus pacientes una sesión anual con MFM.

La estrategia de liberación de tejidos blandos no es excepcional en el manejo quirúrgico de la PCI. Las resecciones de la unión miofascial o de las fascias se usan habitualmente en la terapia quirúrgica multinivel estándar de la espasticidad $[11,12]$. Adicionalmente, técnicas mínimamente invasivas sobre los tejidos blandos están apareciendo como alternativas a intervenciones más agresivas. Kondo et al describieron, en un ensayo clínico prospectivo, los resultados positivos de la 'cirugía de liberación muscular selectiva', que se basa en cortes intramusculares para alargar los grupos musculares [13]. Mitsiokapa et al publicaron un estudio retrospectivo que incidía en efectos positivos del 'alargamiento miofascial percutáneo selectivo' de los aductores de la cadera o de los isquiotibiales asociado al bloqueo alcohólico del obturador [14].

A pesar de que los desarrolladores de la MFM mantienen que 'han realizado al menos 23.000 intervenciones' [15], no existen estudios que hayan evaluado la eficacia y la seguridad de la MFM. La participación de resultados en las comunidades científica y clínica internacionales por parte de sus cirujanos es escasa, no hay registro sobre el desarrollo inicial de la técnica, no hay datos sobre los efectos adversos, su coste (inicialmente 6.000 euros) es muy alto considerando su supuesta simplicidad, y la plausibilidad biológica de los mecanismos de acción defendidos por los desarrolladores de la técnica es baja. Esto hace que la MFM sea rechazada por parte de la comunidad médica. A pesar de todo ello, los pacientes con PCI y otros tipos de espasticidad y sus familias continúan solicitando la realización de MFM, y tanto los pacientes como sus médicos solicitan investigación e información objetivas sobre esta técnica.

Considerando que la indicación de la técnica está sin definir y sin regular actualmente, se requiere un estudio observacional como paso previo a la realización de estudios de diseño superior (ensayos clínicos y cohortes con criterios de selección bien definidos con un grupo control tratado con las técnicas habituales). Por ello, el objetivo de este estudio es explorar los cambios funcionales y en la marcha tras una sola sesión de MFM en escolares con PCI espástica bilateral con afectación predominante de los miembros inferiores. Queremos enfatizar que no estuvimos implicados en la decisión de los padres de optar por la MFM ni participamos en la indicación de los movimientos quirúrgicos, siendo la única implicación del equipo investigador la evaluación previa y posterior a la MFM.

\section{Pacientes y métodos}

\section{Participantes}

Diseñamos un estudio prospectivo observacional autocontrolado para comparar la situación clínica pre y post-MFM, que fue aprobado por nuestro comité de ética (CEIC Universidad Autónoma de Madrid). Se reclutaron 25 escolares afectados por PCI cuyos padres o tutores hubieran decidido previamente realizar una MFM. Los padres tomaron esa decisión de manera unilateral en función de publicidad a través de Internet o de la experiencia de otros padres, pero siempre fuera de la indicación clínica y en contra de la opinión de su médico habitual. Sin embargo, para monitorizar cuidadosamente a los pacientes, sus médicos ofrecieron a los padres la inclusión en este estudio. La selección ocurrió entre junio de 2006 y junio de 2007 en tres centros (Hospital Universitario La Paz, Hospital Universitario Gregorio Marañón y Hospital Universitario Niño Jesús), y a través de las asociaciones de padres con hijos con PCI. No se realizó el precálculo del tamaño muestral por la naturaleza exploratoria del estudio y la imposibilidad de prever el número de pacientes que iban a ser intervenidos. Los criterios de inclusión en el estudio (distintos de los usados para la realización de MFM) fueron: PCI espástica bilateral simétrica con afectación predominante de los miembros inferiores, Gross Motor Function Classification System (GMFCS) I-IV, Manual Ability Classification System I-II, Communication Function Classification System I, capacidad de caminar siete metros independientemente o con asistencia, ausencia de tratamiento oral para la espasticidad en el momento del tratamiento o en el seguimiento, no realización de tratamientos ortopédicos convencionales en el año previo, ausencia de infiltraciones con toxina botulínica en los seis meses previos y ambiente familiar cooperativo. El criterio de exclusión incluye la indicación de cirugía multinivel tras la primera evaluación en nuestro grupo.

\section{Variables del estudio}

Los pacientes fueron evaluados en el mes previo a la cirugía (mediana: 12,5 días; rango: 1-29 días) y de 3 a 7 meses después (mediana: 4 meses; rango: 3-7 meses). No fue necesaria la espera de un año para 
realizar la evaluación posquirúrgica (habitual en la cirugía ortopédica multinivel convencional) debido a la naturaleza menos agresiva de la intervención.

Los pacientes guardaron reposo en cama durante una semana y recuperaron la fisioterapia al mes de la cirugía.

Se midieron tres escalas de funcionalidad en ambas visitas: GMFCS [16], dimensión E de la Gross Motor Function Measure (GMFM66E) [17] y Functional Mobility Scale (FMS) [18]. También se realizó un análisis instrumental de marcha en un laboratorio equipado por un sistema Codamotion (Charnwood Dynamics Ltd.), posicionando los marcadores según los modelos intersegmentarios. Tras varias adquisiciones de entrenamiento, se grabó un mínimo de 15-20 adquisiciones, aunque sólo los 4-5 ciclos de marcha con mayor calidad técnica se seleccionaron para el promedio. Se calculó la media de 16 parámetros de marcha izquierdos y 16 derechos [19] de esos ciclos, y se normalizó (Z-score) respecto a una base de datos de referencia de 27 escolares sanos [20]. En la segunda visita, se solicitó de los padres la copia del mapa topográfico de los cortes.

\section{Análisis estadístico}

Dado que la MFM es una intervención multinivel con diferentes grados de intervención en la cabeza, miembros superiores, tronco y miembros inferiores, se investigó mediante análisis de conglomerados jerárquicos la posibilidad de que se hubieran aplicado patrones repetidos de cortes o, por el contrario, que fueran aleatorios según el paciente. Los patrones de intervención y los grupos de pacientes se definieron mediante distancias binarias, tomando la media como criterio de agrupación, y los resultados se representaron en un heatmap (Fig. 1). Adicionalmente, se realizó un test exacto de Fisher para saber si los patrones de intervención dependían del valor prequirúrgico del GMFCS.

Para comparar los valores pre y post-MFM de las escalas clínicas se realizaron tests de Wilcoxon para datos pareados. En paralelo, se realizó un estudio secundario utilizando los operadores 'mejora clínicamente significativa, 'sin cambios' o 'empeoramiento clínicamente significativo' para reflejar mejor el resultado clínico por paciente. Los operadores significativos fueron definidos siguiendo un consenso dentro del equipo investigador -cualquier cambio en el GMFCS, un aumento o disminución igual o mayor del 5\% en la GMFM66E y cualquier cambio en la FMS (Fig. 2a)-. Se realizó después un análisis de correspondencias múltiples para explorar la relación entre los cambios funcionales (va-
Figura 1. Estudio de los patrones de cortes musculares realizados por los cirujanos. El heatmap muestra los músculos seccionados durante la miofibrotomía múltiple de cada niño. Los cortes musculares se representan en las filas y se enumeran en el lado derecho de la figura. Los códigos de cada paciente se representan en la parte inferior de la figura. Cada cuadrado es la interacción entre el paciente y la localización del corte. El color negro se usa si un músculo ha sido cortado en un paciente específico. El dendrograma de la parte superior del heatmap representa la clasificación jerárquica de cada paciente. El dendrograma de la izquierda representa la clasificación de la localización del corte. En ambos, cuanto más alejado esté el nodo entre dos variables, menor asociación existe entre ellas. Se observa que los patrones de cortes no son uniformes entre los pacientes y no todos los músculos se cortan con la misma frecuencia. El análisis de conglomerados muestra dos grandes grupos de músculos, uno de ellos de los músculos frecuentemente intervenidos y otro de aquéllos que rara vez se intervienen. Entre los que se cortan con frecuencia se observan tres grupos de músculos que suelen intervenirse de manera conjunta, señalados como A, B y C. El análisis demuestra tres patrones de intervención en los pacientes. El patrón 1 (pacientes 17, 13, 20, 9, 10, 11, 6 y 5) se caracteriza, principalmente, por la intervención del grupo A con algunos cortes de los músculos del grupo B y C. El patrón 2 (pacientes 15, 22, 16, 2, 7, 21, 8, 3 y 1) es una combinación de los cortes del grupo $C$ mayoritariamente con algunos de los otros grupos. El patrón 3 (pacientes 1, 14, 18 y 12) combina los cortes realizados en el grupo B con pocos cortes en los otros grupos, además de con cortes en los músculos que rara vez son intervenidos.

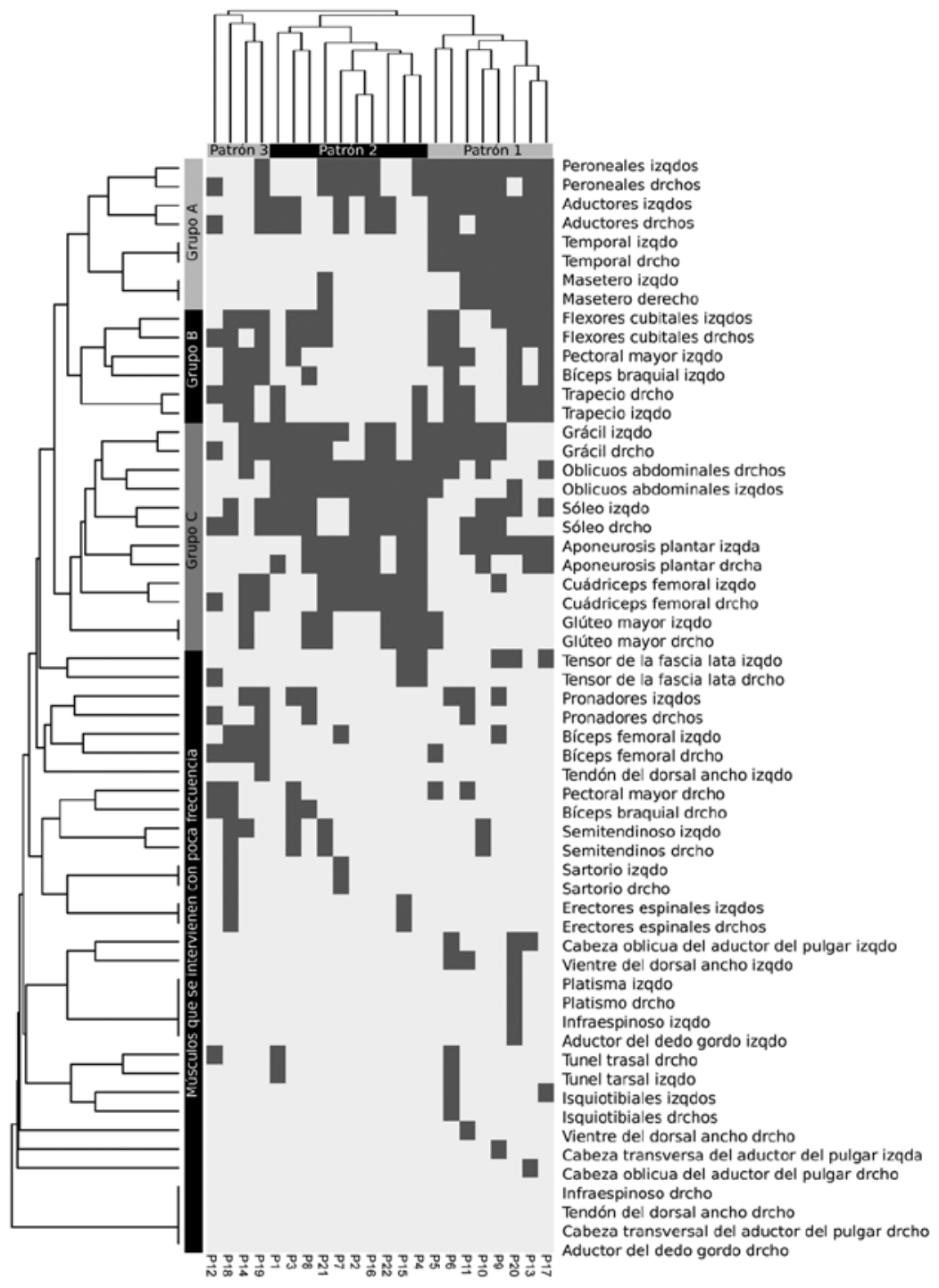


Figura 2. a) Cambios en el estado funcional tras una miofibrotomía múltiple: distribución relativa de las diferencias postratamiento en las escalas funcionales; b) Representación gráfica de las dos primeras dimensiones definidas en el análisis de correspondencias múltiples. Las escalas funcionales se representan con triángulos: (+) a su lado indica mejoría, (=) indica sin cambios y (-) indica empeoramiento. Los círculos 'TIPO1', 'TIPO2' y 'TIPO3' indican los patrones de cortes musculares descritos en la figura 1. Cuanto más cerca estén de los triángulos, más pacientes muestran esos cambios funcionales a la vez. Los puntos que representan 'mejoras clínicamente significativas' tienden a estar en el lado izquierdo del gráfico, mientras que los de 'empeoramiento clínicamente significativo' están en el cuadrante superior derecho. Los puntos que representan a los patrones tipo 1 y tipo 2 se sitúan cerca del origen de coordenadas, lo que indica escasa correlación de estos patrones con los cambios funcionales. Sin embargo, el punto que representa al patrón tipo 3 se sitúa cercano a los triángulos relacionados con 'empeoramiento clínicamente significativo', lo que indica una probable relación con peores resultados funcionales.

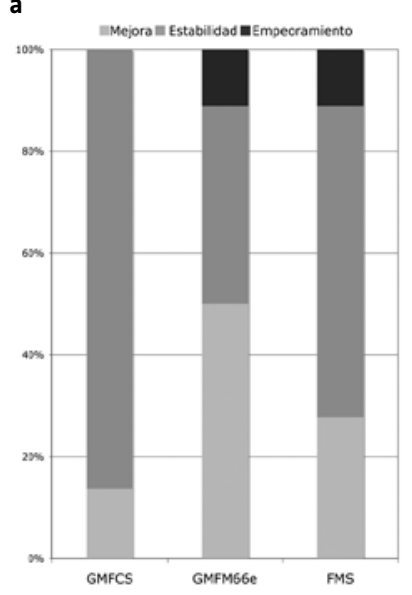

b

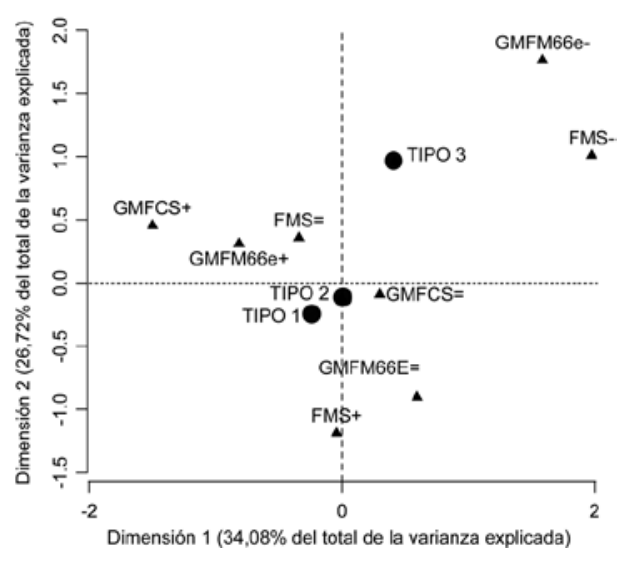

riables activas) y los patrones de corte en la MFM (factor suplementario) (Fig. 2b).

Como medida de normalización en la marcha, se calcularon las diferencias entre los valores absolutos del Z-score en cada parámetro de marcha (pre menos post-MFM). Los intervalos de confianza al 95\% de estas diferencias se estimaron mediante el intervalo de confianza bootstrap acelerado y corregido para sesgos (1.000 repeticiones). No se realizaron correcciones estadísticas por comparaciones múltiples debido a la naturaleza exploratoria del estudio. Los resultados se mostraron en un gráfico de Forest (Fig. 3).

Se utilizó el análisis por protocolo en todas las comparaciones y el sistema estadístico $R$.

\section{Resultados}

\section{Descripción de los pacientes}

Tres pacientes no acudieron a la segunda visita por motivos personales. Veintidós pacientes (15 niños y siete niñas) se incluyeron en el análisis final (edad mediana: 9 años y 6 meses; Q1-Q3: 7 años y 1 mes a 11 años y 6 meses. Talla mediana: $130 \mathrm{~cm}$; Q1-Q3: 121-140 cm. Peso mediano: 30 kg; Q1-Q3: 24-40 kg). Siete pacientes se sometieron a este tipo de intervención por primera vez y los otros 15 habían sido intervenidos previamente, pero hacía más de un año. La tabla muestra la situación funcional de los niños seleccionados previa a la MFM. La mayoría de los niños estaba moderadamente afecta, con un GMFCS de II o III y un GMFM66E entre el 20 y el $80 \%$, pero con unos valores variables en la FMS.

\section{Patrones de corte muscular}

La figura 1 es un heatmap que expresa los patrones de corte de la MFM sobre los músculos. Los patrones de corte no fueron uniformes entre los pacientes y no todos los músculos se cortaron con la misma frecuencia. El análisis de conglomerados jerárquicos reveló dos grandes grupos de músculos en función de la frecuencia con la cual los cirujanos intervinieron sobre ellos: un grupo con músculos donde se intervenía frecuentemente y un grupo donde se intervenía pocas veces. Entre los músculos que se intervinieron de forma frecuente, se identificaron tres subgrupos de músculos. El subgrupo A está formado por los músculos peroneos izquierdos y derechos, los aductores izquierdos y derechos, los temporales izquierdos y derechos, y los maseteros izquierdos y derechos. El subgrupo B clasificó conjuntamente a los flexores cubitales del carpo y los flexores superficiales de los dedos izquierdos y derechos, el pectoral mayor izquierdo, el bíceps braquial izquierdo y los trapecios derecho e izquierdo. El subgrupo $\mathrm{C}$ es un conglomerado formado por los gráciles izquierdos y derechos, los oblicuos abdominales izquierdos y derechos, los sóleos izquierdo y derecho, las aponeurosis plantares izquierda y derecha, los cuádriceps femorales izquierdo y derecho, y los glúteos mayores izquierdo y derecho.

El análisis jerárquico también definió tres patrones de intervención en los pacientes en función de los grupos musculares donde se intervino (Tabla). El patrón 1 (pacientes 17, 13, 20, 9, 10, 11, 6 y 5) consistió en una combinación de cortes, principalmente del subgrupo A, de músculos con pocos cortes en los subgrupos B y C. El patrón 2 (pacientes $15,22,16,2,7,21,8,3$ y 1) fue una combinación de cortes, principalmente en el subgrupo de músculos $\mathrm{C}$, con pocos cortes en los otros dos subgrupos. El patrón 3 (pacientes 19, 14, 18 y 12) fue una combinación de cortes en el subgrupo muscular B, con 
pocos cortes en los otros dos subgrupos y con cortes en los músculos que eran poco seleccionados.

No se detectó correlación entre el GMFCS prequirúrgico y el patrón de corte muscular que se aplicaba a los pacientes (test exacto de Fisher, $p=0,391$ ).

\section{Cambios en las escalas funcionales tras la MFM}

La tabla muestra la distribución de los datos de las escalas funcionales en ambas visitas y el cambio observado tras el tratamiento. Se detectaron cambios estadísticamente significativos en el GMFM66E (intervalo de confianza al 95\% $=0-6,94 \%, p=0,044$ ), con una suave tendencia a mejorar en el GMFCS $(p=0,083)$ y en el ítem 2 de la FMS $(p=0,066)$. La figura 2a muestra los resultados del estudio de evaluación secundaria de la distribución de los cambios en el GMFCS, la GMFM66E y la FMS definidos en cada paciente como 'mejoras clínicamente significativas', 'sin cambios' o 'empeoramiento clínicamente significativo'. Aunque la mayoría de los pacientes mantuvieron su estado funcional, dos pacientes empeoraron en la GMFM66E. La figura 2b muestra los resultados del análisis de correspondencias múltiples que relaciona los cambios en las escalas funcionales con el patrón de cortes musculares empleado en cada paciente. Como se puede observar, el patrón 3 de intervención parece relacionarse con el empeoramiento de las escalas funcionales, mientras que los patrones 1 y 2 no se correlacionan demasiado con los cambios en las escalas funcionales.

\section{Cambios en la marcha de los pacientes tras la MFM}

La figura 3 muestra el análisis de las variables de marcha normalizadas tras la MFM. El efecto en los parámetros de marcha a la MFM es muy variable. Sólo cinco de las 32 variables de marcha estaban más cerca de la normalidad de manera estadísticamente significativa: rango de flexión de la cadera izquierda, dorsiflexión máxima del tobillo izquierdo en el apoyo, normalización de la velocidad de la marcha del lado derecho, rotación media de la pelvis derecha en el apoyo y máxima dorsiflexión del tobillo derecho en la fase de vuelo. Otros parámetros de marcha mostraban una tendencia a la mejora, por ejemplo, la dorsiflexión máxima del tobillo derecho en el apoyo o la flexión de la rodilla derecha en el contacto inicial. Sin embargo, también hay variables de marcha que se alejan de la normalidad tras la MFM, como la inclinación pélvica media derecha e izquierda, la flexión mínima de la cadera derecha e izquierda o el rango de flexión de ambas rodillas.
Figura 3. Gráficos de Forest que indican la normalización de los parámetros de marcha izquierdos y derechos. Los parámetros y sus unidades se indican en el centro del gráfico. Se utilizó la diferencia entre el valor absoluto del Z-score pretratamiento menos el valor absoluto del Z-score postratamiento como indicador de la normalización. Una normalización positiva indica que el parámetro de marcha estaría más cerca de la base de datos de referencia tras el tratamiento. El valor 0 indica la ausencia de cambio. Cada cuadrado representa el valor medio de normalización para cada parámetro, y cada línea representa su intervalo de confianza al 95\%. Sólo cinco de los 32 parámetros de marcha mostraron una normalización estadísticamente significativa (rango de flexión de la cadera izquierda, dorsiflexión máxima en el apoyo izquierda, velocidad normalizada derecha, rotación pélvica media derecha en el apoyo y dorsiflexión máxima en la fase oscilante derecha).

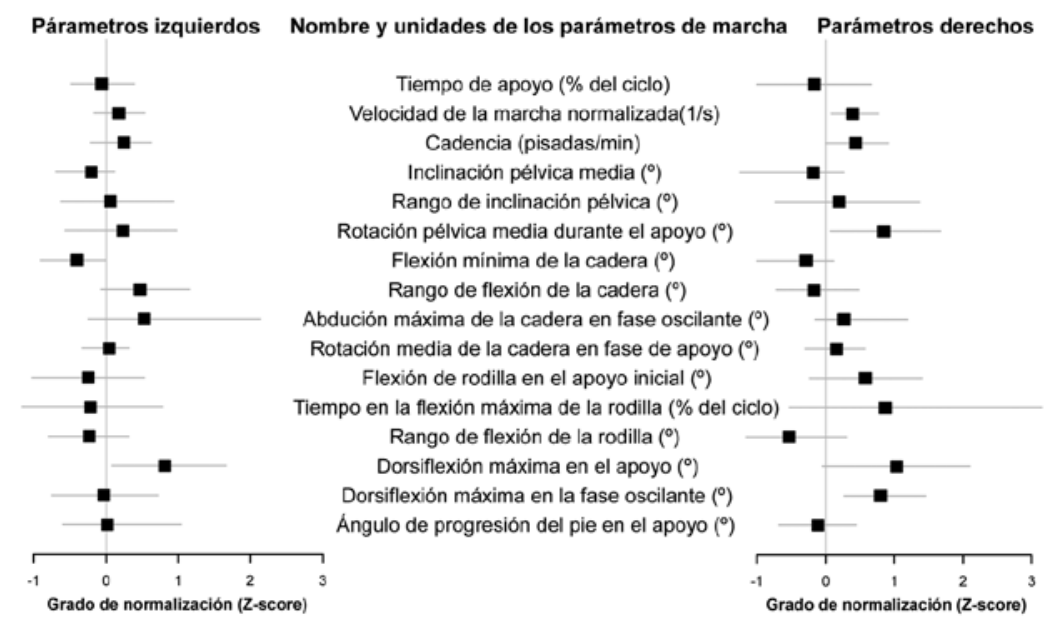

\section{Discusión}

Éste es el primer y único estudio independiente y objetivo que evalúa el efecto de la MFM en las escalas funcionales y en los parámetros de marcha en una cohorte de escolares con diplejía espástica de afectación predominante en los miembros inferiores y que conservan la capacidad para deambular.

La falta de aleatorización y control del brazo de tratamiento es una de las debilidades de este estudio; sin embargo, un diseño diferente no hubiese sido ético por el equilibrio riesgo/beneficio que presentaba esta técnica en el momento del diseño del estudio. Además, los examinadores no podían ser ciegos, pero, sin embargo, la independencia del equipo investigador y la ausencia de conflictos de interés solventan, en cierta parte, estos problemas metodológicos.

Uno de los inconvenientes para la realización del estudio ha sido la falta de protocolización de los cortes realizados por los cirujanos de Ulzibat, lo que ha hecho necesario emplear técnicas de matemáticas para descubrir la existencia de patrones de intervención en los distintos pacientes. 
Tabla. Cambios en las escalas funcionales tras la miofibrotomía múltiple. La dimensión E de la Gross Motor Function Measure (GMFM66E) se divide en intervalos subjetivos del 20\% para expresar la distribución de los datos. El cambio se expresa como la diferencia entre el valor pretratamiento y el valor postratamiento. Un valor positivo indica mejoría. La GMF66E es el único parámetro que muestra cambios estadísticamente significativos. Los cambios de la FMS, ítem 2, no son significativos, pero muestran tendencia a mejorar.

\begin{tabular}{llllll}
\hline & GMFCS & GMFM66E & FMS ítem 1 & FMS ítem 2 & FMS ítem 3 \\
\hline $\begin{array}{lllll}\text { Situación pretratamiento } \\
\text { (n.o de pacientes y }\end{array}$ & I: $2(9,1 \%)$ & $<20 \%: 2(11,1 \%)$ & $1: 3(16,7 \%)$ & $1: 3(16,7 \%)$ & $1: 5(27,8 \%)$ \\
porcentaje) & II: $7(31,8 \%)$ & $20-40 \%: 4(22,2 \%)$ & $2: 1(5,6 \%)$ & $2: 4(22,2 \%)$ & $2: 3(16,7 \%)$ \\
& III: $10(45,5 \%)$ & $40-60 \%: 4(22,2 \%)$ & $3: 4(22,2 \%)$ & $3: 2(11,1 \%)$ & $3: 1(5,6 \%)$ \\
& IV: $3(13,6 \%)$ & $60-80 \%: 4(22,2 \%)$ & $4: 0$ & $4: 2(11,1 \%)$ & $4: 7(38,9 \%)$ \\
& & $>80 \%: 3(16,7 \%)$ & $5: 3(16,7 \%)$ & $5: 2(11,1 \%)$ & $5: 2(11,1 \%)$ \\
& NA: 4 & $6: 7(38,9 \%)$ & $6: 5(27,8 \%)$ & $6: 0$ \\
Cambio (mediana) & 0 (rango: 0-1) & $4,86 \%($ rango: $-19,4-11,1)$ & $0($ rango: $1-3)$ & 0 (rango: 0-3) & 0 (rango: 0-3) \\
\hline$p$ & 0,083 & $0,044^{\text {a }}$ & 0,655 & 0,066 & 0,257 \\
\hline
\end{tabular}

FMS: Functional Mobility Scale; GMFCS: Gross Motor Function Classification System; NA: no aplicable. a Test de Wilcoxon.

La selección de las escalas funcionales y de las variables de la marcha (adquiridas mediante análisis computarizado de la marcha) para medir el efecto de la MFM representan dos de las mayores fortalezas del estudio, además de ser complementarias, pues se usan ampliamente para la representación de las alteraciones de la marcha y la discapacidad [2124]. Una ventaja adicional de nuestro estudio es la normalización de los datos de análisis de la marcha mediante nuestro propio grupo de referencia de escolares sanos explorados en el mismo laboratorio y con los mismos métodos que los pacientes sometidos a MFM [20]. No hemos usado el Gillette Gait Index (anteriormente, Normalcy Index) como variable, ya que su uso correcto requiere una muestra de pacientes mayor para tener validez externa [25].

La presencia de tres patrones de cortes diferentes en esta muestra de pacientes necesita contrastarse con los promotores de la técnica. No sabemos si esos patrones de cortes se realizan de manera intencionada como diferentes estrategias de tratamiento multinivel por parte de los cirujanos de Ulzibat, y en nuestro análisis no hemos encontrado claras correlaciones entre el GMFCS prequirúrgico y el patrón de cortes musculares realizados. Sin embargo, este estudio ha demostrado que algunos pacientes de nuestra muestra presentan una mejora de la funcionalidad tras la MFM, mientras que otros no mejoran o incluso empeoran. Los pacientes que recibieron el patrón 3 de intervención presentan un empeoramiento clínico medido por las escalas, mientras que el uso de los otros patrones no se correlaciona con los resultados funcionales.
La cirugía ortopédica convencional y la MFM se utilizan en distintos grupos de pacientes con PCI. La cirugía ortopédica convencional se indica en pacientes con contracturas fijas, articulaciones dislocadas, problemas rotacionales o problemas para el uso de calzado $[11,26]$. Por ello no son comparables los resultados de la MFM con los resultados de series de pacientes tratados con cirugía ortopédica convencional. La toxina botulínica multinivel es también una terapia con pruebas sólidas para su uso en la PCI $[2,27]$, y los pacientes candidatos a este tratamiento podrían ser similares a los de la MFM. Es difícil comparar los resultados de ambos tratamientos sin conocer bien las indicaciones y los protocolos utilizados en la MFM. También existen problemas de ese tipo cuando intentamos comparar los resultados de nuestro estudio con otras técnicas basadas en la liberación de tejidos blandos. Kondo et al estudiaron la efectividad de la 'cirugía de liberación muscular' y observaron un cambio significativo en la dimensión E del GMFM únicamente en los pacientes con GMFCS IV que tardó nueve meses en aparecer [13]. Mitsiokapa et al estudiaron retrospectivamente los resultados tras alargamientos miofasciales percutáneos selectivos (técnica similar a la MFM) junto con bloqueo alcohólico del nervio obturador. A los seis meses del tratamiento, 33 de los 58 niños tratados mejoraron en uno o dos niveles su GMFCS. Estos resultados tan positivos no han sido confirmados por otros estudios [14].

En nuestra cohorte de escolares, definida con criterios de inclusión estrictos, se ha mostrado una mejora estadísticamente significativa en la GMFM66E. 
También hemos observado una mejora significativa en cinco de los 32 parámetros de marcha analizados. Sin embargo, dadas las limitaciones del estudio, estos resultados no pueden fundamentar la incorporación de la técnica a la práctica clínica ni son suficientes para conocer los mecanismos sobre los efectos positivos y negativos de esta intervención.

Aunque el tamaño muestral de este estudio es discutible, esto se ha compensado con estrategias de diseño y análisis estadístico, y con cautela en la interpretación. A pesar de ello, enfatizamos que la MFM debería evitarse fuera de protocolos de investigación por la falta de información sobre su eficacia (en especial, a largo plazo) y porque existen dudas sobre la seguridad de esta técnica, sobre todo una vez demostrado que una de las estrategias de intervención podría ser peligrosa.

Sin embargo, de cara a la realización de nuevos ensayos clínicos de la MFM, el equilibrio riesgo/beneficio ha cambiado. Anteriormente a este trabajo, la inclusión de niños con parálisis cerebral en un ensayo clínico de MFM no era ética, dada la ausencia de información de modelos animales y de plausibilidad biológica, pero, de acuerdo con los resultados de este estudio, podría realizarse un ensayo clínico aleatorizado con un seguimiento a largo plazo para comprobar la seguridad, la eficacia y los efectos secundarios de manera controlada.

\section{Bibliografía}

1. Platt MJ, Cans C, Johnson A, Surman G, Topp M, Torrioli MG, et al. Trends in cerebral palsy among infants of very low birthweight $(<1500 \mathrm{~g})$ or born prematurely $(<32$ weeks $)$ in 16 European centres: a database study. Lancet 2007; 369: 43-50.

2. Heinen F, Desloovere K, Schroeder AS, Berweck S, Borggraefe I, Van Campenhout A, et al. The updated European Consensus 2009 on the use of botulinum toxin for children with cerebral palsy. Eur J Paediatr Neurol 2010; 14: 45-66.

3. Deon LL, Gaebler-Spira D. Assessment and treatment of movement disorders in children with cerebral palsy. Orthop Clin North Am 2010; 41: 507-17.

4. Oppenheim WL. Complementary and alternative methods in cerebral palsy. Dev Med Child Neurol 2009; 51 (Suppl 4): S122-9.

5. Rosenbaum P. Controversial treatment of spasticity: exploring alternative therapies for motor function in children with cerebral palsy. I Child Neurol 2003; 18 (Suppl 1): S89-94.

6. Liptak GS. Complementary and alternative therapies for cerebral palsy. Ment Retard Dev Disabil Res Rev 2005; 11: 156-63.

7. Weisleder P. Unethical prescriptions: alternative therapies for children with cerebral palsy. Clin Pediatr (Phila) 2010; 49: 7-11.
8 Ulzibat V, Shishov S. Operative treatment of primary fibromyalgia (myofibrillosis). Complement Ther Med 1995; 3: 72-4.

9 'En Rusia se hizo el milagro'. URL: http://es.wikipedia.org/wiki/ ulzibat. [02.10.2012].

10 Econ Medical Services. URL: http://www.serviciosmedicosecon com/eng/index.html. [02.12.2012].

11 Gage JR. The identification and treatment of gait problems in cerebral palsy. 2 ed. London: Mac Keith Press; 2009.

12. Abel MF, Damiano DL, Pannunzio M, Bush J. Muscle-tendon surgery in diplegic cerebral palsy: functional and mechanical changes. J Pediatr Orthop 1999; 19: 366-75.

13. Kondo I, Hosokawa K, Iwata M, Oda A, Nomura T, Ikeda K, et al. Effectiveness of selective muscle-release surgery for children with cerebral palsy: longitudinal and stratified analysis. Dev Med Child Neurol 2004; 46: 540-7.

14. Mitsiokapa EA, Mavrogenis AF, Skouteli H, Vrettos SG, Tzanos G, Kanellopoulos AD, et al. Selective percutaneous myofascial lengthening of the lower extremities in children with spastic cerebral palsy. Clin Pediatr Med Surg 2010; 27: 335-43.

15. Institut of Clinical Rehabilitology. Regional patners. URL: http://ulzibat.ru/en/partners/. [02.12.2012].

16. Wood E, Rosenbaum P. The gross motor function classification system for cerebral palsy: a study of reliability and stability over time. Dev Med Child Neurol 2000; 42: 292-6.

17. Avery LM, Russell DJ, Raina PS, Walter SD, Rosenbaum PL. Rasch analysis of the Gross Motor Function Measure: validating the assumptions of the Rasch model to create an intervallevel measure. Arch Phys Med Rehabil 2003; 84: 697-705.

18. Graham HK, Harvey A, Rodda J, Nattrass GR, Pirpiris M. The Functional Mobility Scale (FMS). J Pediatr Orthop 2004; 24: 514-20.

19. Schutte LM, Narayanan U, Stout JL, Selber P, Gage JR, Schwartz MH. An index for quantifying deviations from normal gait. Gait Posture 2000; 11: 25-31.

20 Pulido-Valdeolivas I, Gómez-Andrés D, Martín-Gonzalo JA, López-López J, Gómez-Barrena E, Sánchez-Hernández JJ, et al. Gait parameters in a reference sample of healthy Spanish schoolchildren: multivariate descriptive statistics and asymmetries observed in left and right cycles. Neurologia 2013; 28: 145-52.

21. Cretual A, Bervet K, Ballaz L. Gillette Gait Index in adults. Gait Posture 2010; 32: 307-10.

22 Gray L, Ng H, Bartlett D. The gross motor function classification system: an update on impact and clinical utility. Pediatr Phys Ther 2010; 22: 315-20.

23. Russell DJ, Avery LM, Walter SD, Hanna SE, Bartlett DJ, Rosenbaum PL, et al. Development and validation of item sets to improve efficiency of administration of the 66-item Gross Motor Function Measure in children with cerebral palsy. Dev Med Child Neurol 2010; 52: e48-54.

24. Harvey AR, Morris ME, Graham HK, Wolfe R, Baker R. Reliability of the functional mobility scale for children with cerebral palsy. Phys Occup Ther Pediatr 2010; 30: 139-49.

25. Tulchin K, Campbell S, Browne R, Orendurff M. Effect of sample size and reduced number of principle components on the Gillette Gait Index. Gait Posture 2009; 29: 526-9.

26. Lynn AK, Turner M, Chambers HG. Surgical management of spasticity in persons with cerebral palsy. PM R 2009; 1: 834-8.

27. Unlu E, Cevikol A, Bal B, Gonen E, Celik O, Kose G. Multilevel botulinum toxin type a as a treatment for spasticity in children with cerebral palsy: a retrospective study. Clinics (Sao Paulo) 2010; 65: 613-9. 
External evaluation of gait and functional changes after a single-session multiple myofibrotenotomy in school-aged children with spastic diplegia

Introduction. About 23,000 patients with spasticity voluntarily chose to undergo a multiple myofiberotomy (MMF), which is an alternative technique consisting in sectioning soft tissues in order to relieve restrictions in joint movements. This technique, first employed by Ulzibat (Russia), is performed outside orthodox clinical controls.

Aims. To perform an external evaluation of the effects of MMF on motor functionality and gait.

Patients and methods. The study was self-controlled and observation-based and was designed to evaluate the changes in functional variables -Gross Motor Function Classification System, E-dimension of the Gross Motor Function Measure (GMFM) and the Functional Mobility Scale- and 32 gait parameters (measured using analytical instruments) in 22 schoolchildren with spastic diplegia (mean age: 9 years and 6 months; Q1-Q3: 7 years and 11 months to 11 years and 6 months) whose parents opted for an MMF (median of observation: 4 months; range: 3-7 months).

Results. The analysis of hierarchical conglomerates used to determine topographic cut patterns in patients revealed that the surgeons applied one of three sets of cuts to each patient. On analysing the three groups of patients, it was observed that one group worsened overall and another underwent a general significant improvement in the E-dimension of the GMFM (difference of median: $4.86 \% ; 95 \%$ confidence interval $=0-6.94 \%$ ) after the MMF. Some of the gait parameters became significantly normalised (left: hip-flexion range, maximum dorsiflexion with support; right: speed, mean pelvic rotation with support and maximum dorsiflexion with support).

Conclusions. These findings do not back up or justify the use of MMF as a therapeutic option to treat spasticity. It has to be stressed that this technique must be avoided outside study protocols. Nevertheless, results do lay an objective base that may justify a clinical trial and long-term observation-based studies.

Key words. Cerebral palsy. Gait. Surgery. Ulzibat. Walking. 